\title{
Synchronization of Complex Networks with Coupling Delays via Adaptive Pinning Intermittent Control
}

\author{
Hai-Yi Sun ${ }^{1} \quad$ Ning Li $^{2} \quad$ De-Ping Zhao ${ }^{1} \quad$ Qing-Ling Zhang ${ }^{2}$ \\ ${ }^{1}$ College of Science, Shenyang Jianzhu University, Shenyang 110168, China \\ ${ }^{2}$ Institute of Systems Science, Northeastern University, Shenyang 110819, China
}

\begin{abstract}
The problem of exponential synchronization for a class of general complex dynamical networks with nonlinear coupling delays by adaptive pinning periodically intermittent control is considered in this paper. We use the methods of the adaptive control, pinning control and periodically intermittent control. Based on the piecewise Lyapunov stability theory, some less conservative criteria are derived for the global exponential synchronization of the complex dynamical networks with coupling delays. And several corresponding adaptive pinning feedback synchronization controllers are designed. These controllers have strong robustness against the coupling strength and topological structure of the network. Using the delayed nonlinear system as the nodes of the networks, a numerical example of the complex dynamical networks with nonlinear coupling delays is given to demonstrate the effectiveness of the control strategy.
\end{abstract}

Keywords: Complex networks, exponential synchronization, delay, adaptive pinning intermittent control, piecewise Lyapunov stability

\section{Introduction}

Complex networks have sprung up in recent 10 years, presently permeating in various fields of sciences, from physics science to biological science, even to social science, mathematics and engineering ${ }^{[1-5]}$. A complex network which is a typical nonlinear system is composed of a large set of interconnected nonlinear nodes, where the nodes and connections can represent anything. Complex networks have applications in almost all the fields in the real world including the world wide web, the internet, the food webs, the communication networks, the neural networks, the cellular and metabolic networks, the electrical power grids, the social networks, etc ${ }^{[1,2]}$.

There has been a rich body of literatures on analyzing complex networks. And one of the most significant dynamical behaviors that has been widely investigated is the synchronization of its dynamical elements ${ }^{[5-22]}$. In fact, synchronization is a well-established concept and it is typical in the basic motions of nature. There are many interesting synchronization phenomena, e.g., fireflies flashing in unison, crickets chirping in synchrony and heart cells beating in rhythm. And some synchronization phenomena are very useful for us, such as the synchronous transfer of digital and analog signals in communication networks ${ }^{[5]}$. Many control techniques, including linear feedback control ${ }^{[7]}$, adaptive feedback control ${ }^{[8-12]}$, impulsive control ${ }^{[13]}$, pinning control ${ }^{[14,16,18]}$, sliding mode control ${ }^{[19-21]}$ and fuzzy control ${ }^{[22]}$, have been developed to drive the synchronization and anti-synchronization.

On the other hand, intermittent control has a wide variety of applications, such as ecosystem management, wind-

\footnotetext{
Manuscript received February 14, 2013; revised June 6, 2013 This work was supported by National Natural Science Foundation of China (No. 61273008), Science Research Project of Liaoning Provincial Education Department (No. L2012208) and Science Foundation of Ministry of Housing and Urban-Rural Development (No. 2013-K5$2)$.
}

shield wipers intermittent control, and control of hyperuricacidemia control in the treatment of gout ${ }^{[23]}$. An essential benefit of the intermittent control approach may lie in that it has a nonzero control width, and it is easy to be implemented in practical applications. In [15], intermittent control was first introduced to control nonlinear dynamical systems. Since then, many interesting results have been presented for stabilization and synchronization of complex network systems with or without time delays by periodically intermittent control ${ }^{[16-19]}$. Yang and Cao ${ }^{[16]}$ studied the exponential stochastic synchronization problem for coupled neural networks with stochastic noise perturbations. More recently, synchronization of delayed complex dynamical networks by linear periodically intermittent control was also reported ${ }^{[17,18]}$. But the two restrictive conditions that the control width should be greater than the time delays and the time delays should be smaller than the noncontrol width are imposed. Cai et al. ${ }^{[19]}$ further investigated pinning synchronization of complex network with delayed dynamical nodes via linear periodically intermittent control. The results removed the traditional assumptions on control width and time delays mentioned above. Unfortunately, the obtained control gain may be much larger than needed. Therefore, the study of the periodically intermittent control needs further exploration and improvement.

Motivated by the above discussions, this paper aims to handle the problem of exponential synchronization for the complex dynamical networks with nonlinear coupling delays via adaptive pinning periodically intermittent control. Based on the piecewise Lyapunov stability theory combined with adaptive control, pinning control and periodically intermittent control techniques, an improved synchronization criterion and the corresponding adaptive pinning periodically intermittent controllers which are less costly and more useful in practice are proposed. It should be pointed out that the coupling configuration matrices are not assumed to be symmetrical or irreducible. And the aforementioned 
two restrictive conditions in $[16-18]$ are not required. $\mathrm{Nu}-$ merical examples are presented to show the effectiveness of the proposed method.

The rest of the paper is organized as follows. In Section 2, the model, some preliminaries and assumptions are given. In Section 3, exponential synchronization of complex dynamical networks with nonlinear coupling delays via adaptive pinning periodically intermittent control are designed. In Section 4, a numerical example of delayed complex networks are given to demonstrate the effectiveness of the proposed controllers. Conclusions are given in Section 5 .

\section{Model and preliminaries}

The complex dynamical networks with nonlinear coupling delays can be described as

$$
\begin{aligned}
\dot{x}_{i}(t)= & f\left(x_{i}(t), t\right)+\sum_{j=1}^{N} a_{i j} H_{0}\left(x_{j}(t)\right)+ \\
& \sum_{j=1}^{N} b_{i j} H_{1}\left(x_{j}(t-\tau)\right)+u_{i}(t)
\end{aligned}
$$

where $i=1, \cdots, N, x_{i}(t)=\left(x_{i 1}(t), x_{i 2}(t), \cdots, x_{i n}(t)\right)^{\mathrm{T}} \in$ $\mathbf{R}^{n}$ and $u_{i}(t) \in \mathbf{R}^{n}$ are the state vector and the input vector of node $i$ at time $t$, respectively. $f: \mathbf{R}^{n} \times \mathbf{R}^{+} \rightarrow \mathbf{R}^{n}$ is a continuously differentiable vector function. And the nonlinear coupling function $H_{l}(\cdot): \mathbf{R}^{n} \rightarrow \mathbf{R}^{n}$ (where $l=0$, $1)$ is continuous and has the form: $H_{0}\left(x_{j}(t)\right)=\left(h_{01}\left(x_{j 1}(t)\right)\right.$, $\left.h_{02}\left(x_{j 2}(t)\right), \cdots, h_{0 n}\left(x_{j n}(t)\right)\right)^{\mathrm{T}}, H_{1}\left(x_{j}(t-\tau)\right)=\left(h_{11}\left(x_{j 1}(t-\right.\right.$ $\left.\tau)), h_{12}\left(x_{j 2}(t-\tau)\right), \cdots, h_{1 n}\left(x_{j n}(t-\tau)\right)\right)^{\mathrm{T}}, \tau$ is the coupling delay. $A=\left(a_{i j}\right) \in \mathbf{R}^{N \times N}$ and $B=\left(b_{i j}\right) \in \mathbf{R}^{N \times N}$ are the coupling matrices with zero-sum rows, which represent the coupling strength and the underlying topology for the nondelayed configuration and the delayed configuration $\tau$ at time $t$, respectively. $a_{i j} \geqslant 0, b_{i j} \geqslant 0$ for $i \neq j, a_{i j}, b_{i j}$ are defined as follows: If there is a connection from node $j$ to node $i(i \neq j)$, then $a_{i j}>0$ and $b_{i j}>0$, otherwise $a_{i j}=0$ and $b_{i j}=0(i \neq j)$.

When the delayed dynamical network (1) achieves synchronization, the states $x_{i}(t)(i=1,2, \cdots, N)$ will infinitely tend to $s(t)$ as $t \rightarrow \infty$, where $s(t) \in \mathbf{R}^{n}$ is a solution of an isolate node, i.e.,

$$
\dot{s}(t)=f(s(t), t)
$$

where $s(t)$ can be an equilibrium point, a nontrivial periodic orbit, or even a chaotic orbit. Let $C\left([-h, 0], \mathbf{R}^{n}\right)$ be the Banach space of continuous functions mapping the interval $[-h, 0]$ into $\mathbf{R}^{n}$ with the norm $\|\phi\|=\sup _{-h \leqslant \theta \leqslant 0}\|\phi(\theta)\|$, where $\|\cdot\|$ is the Euclidean norm. The rigorous mathematical definition of synchronization for the complex dynamical networks with nonlinear coupling delays (1) is introduced as follows.

Definition 1. Let $x_{i}\left(t ; t_{0} ; \phi\right), i=1,2, \cdots, N$ be a solution of the delayed complex dynamical network (1), where $\phi=\left(\phi_{1}^{\mathrm{T}} \text { and } \phi_{2}^{\mathrm{T}}, \cdots, \phi_{N}^{\mathrm{T}}\right)^{\mathrm{T}}, \phi_{i}=\phi_{i}(\theta) \in C\left([-\tau, 0], \mathbf{R}^{n}\right)$ are the initial conditions. If there exist constants $\alpha>0, \lambda>0$ and a nonempty subset $\Lambda \subseteq \mathbf{R}^{n}$, such that $\phi_{i}$ take values in $\Lambda$ and $x_{i}\left(t ; t_{0} ; \phi\right) \in \mathbf{R}^{n}$ for all $t \geqslant t_{0}$ and

$$
\left\|x_{i}\left(t ; t_{0} ; \phi\right)-s\left(t ; t_{0} ; s_{0}\right)\right\| \leqslant \alpha \mathrm{e}^{-\lambda t} \sup _{-\tau \leqslant \theta \leqslant 0}\left\|\phi(\theta)-s_{0}\right\|
$$

then the delayed complex dynamical networks (1) are said to realize exponential synchronization, and $\Lambda \times \Lambda \times \cdots \times \Lambda$ is called the region of synchrony of the delayed complex dynamical networks (1).

Define the error vector by

$$
e_{i}(t)=x_{i}(t)-s(t), \quad i=1,2, \cdots, N
$$

Then, the error system can be described by

$$
\begin{aligned}
\dot{e}_{i}(t)= & f\left(x_{i}(t), t\right)-f(s(t), t)+\sum_{j=1}^{N} a_{i j} W_{0}\left(e_{j}(t)\right)+ \\
& \sum_{j=1}^{N} b_{i j} W_{1}\left(e_{j}(t-\tau)\right)+u_{i}(t)
\end{aligned}
$$

where $W_{l}\left(e_{j}\left(t-\tau_{l}\right)\right)=H_{l}\left(x_{j}\left(t-\tau_{l}\right)\right)-H_{l}\left(s\left(t-\tau_{l}\right)\right), l=0,1$, $\tau_{0}=0, \tau_{1}=\tau$.

To achieve synchronization of the objective (2), we need Assumption 1 and Lemma 1.

Assumption 1. There exist positive constants $L, \underline{w}_{l}, \bar{w}_{l}$ $(l=0,1)$ satisfying

$$
\begin{aligned}
& \left\|f\left(x_{i}(t), t\right)-f\left(y_{i}(t), t\right)\right\| \leqslant L\left\|x_{i}(t)-y_{i}(t)\right\| \\
& \underline{w}_{l}\left\|e_{i}\left(t-\tau_{l}\right)\right\| \leqslant\left\|W_{l}\left(e_{i}\left(t-\tau_{l}\right)\right)\right\| \leqslant \bar{w}_{l}\left\|e_{i}\left(t-\tau_{l}\right)\right\|
\end{aligned}
$$

where $x_{i}(t)$ and $y_{i}(t)$ are time-varying vectors.

Lemma $1^{[12]}$. If $M=\left(m_{i j}\right)_{N \times N}$ is symmetric and $K=$ $\operatorname{diag}\{k_{1}, \cdots, k_{d}, \underbrace{0, \cdots, 0}_{N-d}\}$, where $k_{i}>0, i=1, \cdots, d$ are sufficiently large, then $M-K<0$ is equivalent to $M_{d}<0$, where $M_{d}$ is the minor matrix of $M$ by removing its first $d$ row-column pairs.

In the following, let $\hat{A}^{s}=\frac{\hat{A}+\hat{A}^{\mathrm{T}}}{2}, \lambda_{\max }\left(\hat{A}^{s}\right)$ be the biggest eigenvalue of matrix $\hat{A}^{s}$, where $\hat{A}$ is a modified matrix of $A$ via replacing the diagonal elements $a_{i i}$ by $\left(\frac{\underline{w}_{0}^{2}}{\bar{w}_{0}^{2}}\right) a_{i i}$, $\overline{\hat{A}}_{i j}^{s}=\hat{A}_{d+i, d+j}^{s}$ for $i, j=1, \cdots, N-d, b=\max \left\{\left|b_{i i}\right|\right\}, i=$ $1,2, \cdots, N$

\section{Exponential synchronization of de- layed complex dynamical networks via adaptive pinning periodically in- termittent control}

\subsection{Exponential synchronization of de- layed complex dynamical networks with same nodes}

It is too costly and impractical to add controllers to all nodes in a large-scale network. To reduce the number of controlled nodes, we will introduce several useful adaptive pinning periodically intermittent feedback criteria for the delayed complex dynamical networks (1) in this section. In order to realize synchronization of the delayed complex networks by adaptive pinning periodically intermittent control, some controllers are added to partial nodes of the network. Without loss of generality, if the first $d$ nodes are selected and pinned, then the controllers $u_{i}(t)(1 \leqslant i \leqslant N)$ can be 
described by

$u_{i}(t)=\left\{\begin{array}{l}-k_{i}(t) e_{i}(t), \quad 1 \leqslant i \leqslant d, \quad t \in[\kappa T, \kappa T+h) \\ 0, \quad d+1 \leqslant i \leqslant N, t \in[\kappa T, \kappa T+h) \\ 0, \quad 1 \leqslant i \leqslant N, t \in[\kappa T+h,(\kappa+1) T)\end{array}\right.$

with the updating laws being

$$
\dot{k}_{i}(t)=\alpha_{i} \mathrm{e}^{a_{1} t}\left\|e_{i}(t)\right\|^{2}
$$

where $\alpha_{i}(i=1,2, \cdots, d)$ are positive constants, $T>0$ denotes the control period, $0<h<T$ and $\kappa=0,1,2, \cdots$. Thus, the error system (5) can be rewritten as

$$
\left\{\begin{aligned}
& \dot{e}_{i}(t)= f\left(x_{i}(t), t\right)-f(s(t), t)+\sum_{j=1}^{N} a_{i j} W_{0}\left(e_{j}(t)\right)+ \\
& \sum_{j=1}^{N} b_{i j} W_{1}\left(e_{j}(t-\tau)\right)-k_{i}(t) e_{i}(t) \\
& 1 \leqslant i \leqslant d, \quad t \in[\kappa T, \kappa T+h) \\
& \dot{e}_{i}(t)= f\left(x_{i}(t), t\right)-f(s(t), t)+\sum_{j=1}^{N} a_{i j} W_{0}\left(e_{j}(t)\right)+ \\
& \sum_{j=1}^{N} b_{i j} W_{1}\left(e_{j}(t-\tau)\right), \\
& 1 \leqslant i \leqslant d, \quad t \in[\kappa T, \kappa T+h) \\
& \text { or } t \in[\kappa T+h,(\kappa+1) T) .
\end{aligned}\right.
$$

Based on Assumption 1, synchronization criterion of the controlled delayed complex networks (1) is deduced as follows.

Theorem 1. Under Assumption 1, if there exist positive constants $\varepsilon, a_{1}, a_{2}$ and $\alpha_{i}$, such that

$$
\begin{aligned}
& a_{1}>\frac{N \bar{w}_{1}^{2}}{q} \\
& \left(L+\frac{N b^{2} q}{2}+\frac{1}{2} a_{1}\right) I_{N-d}+\bar{w}_{0}^{2} \overline{\hat{A}}^{s} \leqslant 0 \\
& {\left[L+\frac{N b^{2} q}{2}-\frac{1}{2}\left(a_{2}-a_{1}\right)\right] I_{N}+\bar{w}_{0}^{2} \hat{A}^{s} \leqslant 0} \\
& \varepsilon=\lambda-a_{2}\left(1-\frac{h}{T}\right)>0
\end{aligned}
$$

where $\lambda>0$ is the smallest real root of $a_{1}-\lambda-\frac{N \bar{w}_{1}^{2}}{q} \mathrm{e}^{\lambda \tau}=0$, then the controlled delayed complex networks (1) globally exponentially synchronize with the desired evolution $s(t)$ under the adaptive pinning periodically intermittent controllers (7) and the updating laws (8).

Proof. Construct the following piecewise LyapunovKrasovskii candidate function

$$
V_{\delta}(t)=\frac{1}{2} \sum_{i=1}^{N} e_{i}^{\mathrm{T}}(t) e_{i}(t)+\frac{1}{2} \delta \sum_{i=1}^{N} \mathrm{e}^{-a_{1} t} \frac{\left(k_{i}(t)-k\right)^{2}}{\alpha_{i}}
$$

where

$$
\delta= \begin{cases}1, & t \in[\kappa T, \kappa T+h) \\ 0, & t \in[\kappa T+h,(\kappa+1) T)\end{cases}
$$

and $k$ is an undetermined sufficiently large positive constant. According to Assumption 1, the derivative of $V_{\delta}(t)$ with respect to time $t$ along the solutions of (9) can be calculated as follows.

When $\kappa T \leqslant t<\kappa T+h$, for $\kappa=0,1,2, \cdots$

$$
\begin{aligned}
\dot{V}_{1}(t) & =\sum_{i=1}^{N} e_{i}^{\mathrm{T}}(t) \dot{e}_{i}(t)-\frac{1}{2} a_{1} \sum_{i=1}^{N} \mathrm{e}^{-a_{1} t} \frac{\left(k_{i}(t)-k\right)^{2}}{\alpha_{i}}+ \\
& \sum_{i=1}^{N}\left(k_{i}(t)-k\right) e_{i}^{\mathrm{T}}(t) e_{i}(t)= \\
& \sum_{i=1}^{N} e_{i}^{\mathrm{T}}(t)\left[f\left(x_{i}(t), t\right)-f(s(t), t)\right]+ \\
& \sum_{i=1}^{N} \sum_{j=1}^{N} a_{i j} e_{i}^{\mathrm{T}}(t) W_{0}\left(e_{j}(t)\right)+ \\
& \sum_{i=1}^{N} \sum_{j=1}^{N} b_{i j} e_{i}^{\mathrm{T}}(t) W_{1}\left(e_{j}(t-\tau)\right)- \\
& \sum_{i=1}^{N} k_{i}(t) e_{i}^{\mathrm{T}}(t) e_{i}(t)-\frac{1}{2} a_{1} \sum_{i=1}^{N} \mathrm{e}^{-a_{1} t} \frac{\left(k_{i}(t)-k\right)^{2}}{\alpha_{i}}+ \\
& \sum_{i=1}^{N}\left(k_{i}(t)-k\right) e_{i}^{\mathrm{T}}(t) e_{i}(t) .
\end{aligned}
$$

Let $Q=q I>0$, and we can get

$$
\begin{aligned}
& \sum_{i=1}^{N} \sum_{j=1}^{N} b_{i j} e_{i}^{\mathrm{T}}(t) W_{1}\left(e_{j}(t-\tau)\right) \leqslant \\
& \quad \frac{1}{2} \sum_{i=1}^{N} \sum_{j=1}^{N}\left(b_{i j}\right)^{2} e_{i}^{\mathrm{T}}(t) Q e_{i}(t)+ \\
& \quad \frac{1}{2} \sum_{i=1}^{N} \sum_{j=1}^{N} W_{1}^{\mathrm{T}}\left(e_{j}(t-\tau)\right) Q^{-1} W_{1}\left(e_{j}(t-\tau)\right) \leqslant \\
& \quad \frac{N b^{2}}{2} \sum_{i=1}^{N} e_{i}^{\mathrm{T}}(t) Q e_{i}(t)+\frac{N \bar{w}_{1}^{2}}{2} \sum_{j=1}^{N} e_{j}^{\mathrm{T}}(t-\tau) Q^{-1} e_{j}(t-\tau)= \\
& \quad \frac{N b^{2} q}{2} \sum_{i=1}^{N} e_{i}^{\mathrm{T}}(t) e_{i}(t)+\frac{N \bar{w}_{1}^{2}}{2 q} \sum_{i=1}^{N} e_{i}^{\mathrm{T}}(t-\tau) e_{i}(t-\tau) .
\end{aligned}
$$

Substituting (13) into (12) gives

$$
\begin{aligned}
\dot{V}_{1}(t) \leqslant & \sum_{i=1}^{N} L e_{i}^{\mathrm{T}}(t) e_{i}(t)+ \\
& \sum_{i=1}^{N} \sum_{j=1, j \neq i}^{N} \bar{w}_{0}^{2} a_{i j}\left\|e_{i}(t)\right\|\left\|e_{j}(t)\right\|+ \\
& \sum_{i=1}^{N} \underline{w}_{0}^{2} a_{i i} e_{i}^{\mathrm{T}}(t) e_{i}(t)+\frac{N b^{2} q}{2} \sum_{i=1}^{N} e_{i}^{\mathrm{T}}(t) e_{i}(t)+ \\
& \frac{N \bar{w}_{1}^{2}}{2 q} \sum_{i=1}^{N} e_{i}^{\mathrm{T}}(t-\tau) e_{i}(t-\tau)- \\
& \sum_{i=1}^{N} k e_{i}^{\mathrm{T}}(t) e_{i}(t)-\frac{1}{2} a_{1} \sum_{i=1}^{N} \mathrm{e}^{-a_{1} t} \frac{\left(k_{i}(t)-k\right)^{2}}{\alpha_{i}}= \\
& \bar{e}^{\mathrm{T}}(t)\left[\left(L+\frac{N b^{2} q}{2}+\frac{1}{2} a_{1}\right) I_{N}+\bar{w}_{0}^{2} \hat{A}^{s}-K\right] \bar{e}(t)- \\
& \frac{1}{2} a_{1} \bar{e}^{\mathrm{T}}(t) \bar{e}(t)+\frac{1}{2} \frac{N \bar{w}_{1}^{2}}{q} \bar{e}_{i}^{\mathrm{T}}(t-\tau) \bar{e}_{i}(t-\tau)- \\
& \frac{1}{2} a_{1} \sum_{i=1}^{N} \mathrm{e}^{-a_{1} t} \frac{\left(k_{i}(t)-k\right)^{2}}{\alpha_{i}}
\end{aligned}
$$


where $\bar{e}^{\mathrm{T}}(t)=\left(\left\|e_{1}(t)\right\|_{2},\left\|e_{2}(t)\right\|_{2}, \cdots,\left\|e_{N}(t)\right\|_{2}\right)^{\mathrm{T}}$. Because $k$ is an undetermined sufficiently large positive constant, we can select $k$ as

$$
k>L+\frac{N b^{2} q}{2}+\frac{1}{2} a_{1}+\bar{w}_{0}^{2} \lambda_{\max }\left(\hat{A}^{s}\right) .
$$

Then, we have

$$
\left(L+\frac{N b^{2} q}{2}+\frac{1}{2} a_{1}\right) I_{N}+\bar{w}_{0}^{2} \hat{A}^{s}-K \leqslant 0
$$

where $K=\operatorname{diag}\{k, \cdots, k, \underbrace{0, \cdots, 0}_{N-d}\}$.

Using (16) and condition in the second inequality of (10), combined with Lemma 1, we have

$$
\dot{V}_{1}(t) \leqslant-a_{1} V_{1}(t)+\frac{N \bar{w}_{1}^{2}}{q} V_{0}(t-\tau) .
$$

Similarly, when $\kappa T+h \leqslant t<(\kappa+1) T$, using condition in the third inequality of (10), one has

$$
\begin{aligned}
\dot{V}_{0}(t) \leqslant & \bar{e}^{\mathrm{T}}(t)\left[\left(L+\frac{N b^{2} q}{2}-\frac{1}{2}\left(a_{2}-a_{1}\right)\right) I_{N}+\bar{w}_{0}^{2} \hat{A}^{s}\right] \bar{e}(t)+ \\
& \frac{1}{2}\left(a_{2}-a_{1}\right) \bar{e}^{\mathrm{T}}(t) \bar{e}(t)+\frac{1}{2} \frac{N \bar{w}_{1}^{2}}{q} \bar{e}^{\mathrm{T}}(t-\tau) \bar{e}(t-\tau) \leqslant \\
& \frac{1}{2}\left(a_{2}-a_{1}\right) \bar{e}^{\mathrm{T}}(t) \bar{e}(t)+\frac{1}{2} \frac{N \bar{w}_{1}^{2}}{q} \bar{e}^{\mathrm{T}}(t-\tau) \bar{e}(t-\tau)= \\
& \left(a_{2}-a_{1}\right) V_{0}(t)+\frac{N \bar{w}_{1}^{2}}{q} V_{0}(t-\tau) .
\end{aligned}
$$

According to the first inequality of (10), $a_{1}-\lambda-$ $\frac{N \bar{w}_{1}^{2}}{q} \mathrm{e}^{\lambda \tau}=0$ has a unique positive solution $\lambda>0$ obviously. Take $\bar{Q}_{\delta}=\sup _{-\tau \leqslant s \leqslant 0} V_{\delta}(s)$ and $P_{\delta}(t)=\mathrm{e}^{\lambda t} V_{\delta}(t)$, where $t \geqslant 0$. Apparently, $\bar{Q}_{0} \leqslant \bar{Q}_{1}, P_{0}(t) \leqslant P_{1}(t)$. Let $\Phi(t)=P_{1}(t)-\beta \bar{Q}_{1}$, where $\beta>1$ is a constant. It is easy to see that

$$
\Phi(t)<0, \quad \text { for } \text { all } t \in[-\tau, 0] .
$$

Then, we want to prove that

$$
\Phi(t)<0, \quad \text { for } \text { all } t \in[0, h] .
$$

We adopt the reduction to absurdity. Otherwise, suppose there exists a $t_{0} \in[0, h]$ such that

$$
\begin{gathered}
\Phi\left(t_{0}\right)=0, \quad \dot{\Phi}\left(t_{0}\right) \geqslant 0 \\
\Phi(t)<0, \quad-\tau \leqslant t \leqslant t_{0} .
\end{gathered}
$$

Using (19), (21) and (22), we obtain

$$
\begin{aligned}
& \dot{\Phi}\left(t_{0}\right)=\lambda P_{1}\left(t_{0}\right)+\mathrm{e}^{\lambda t_{0}} \dot{V}_{1}\left(t_{0}\right) \leqslant \\
& \lambda P_{1}\left(t_{0}\right)-a_{1} \mathrm{e}^{\lambda t_{0}} V_{1}\left(t_{0}\right)+ \\
& \frac{N \bar{w}_{1}^{2}}{q} \mathrm{e}^{\lambda t_{0}} V_{0}\left(t_{0}-\tau\right) \leqslant \\
& \left(\lambda-a_{1}\right) P_{1}\left(t_{0}\right)+\frac{N \bar{w}_{1}^{2}}{q} \mathrm{e}^{\lambda \tau} P_{0}\left(t_{0}-\tau\right)< \\
& \left(\lambda-a_{1}\right) \beta \bar{Q}_{1}+\frac{N \bar{w}_{1}^{2}}{q} \mathrm{e}^{\lambda \tau} \bar{Q}_{0}< \\
& \left(\lambda-a_{1}+\frac{N \bar{w}_{1}^{2}}{q} \mathrm{e}^{\lambda \tau}\right) \beta \bar{Q}_{1}=0 .
\end{aligned}
$$

This contradicts the second inequality in (20), and so (19) holds.

Then, we prove that $\Psi(t)=P_{0}(t)-\beta \bar{Q}_{0} \mathrm{e}^{a_{2}(t-h)}<0$ for $t \in[h, T)$. Otherwise, there exists $t_{1} \in[h, T)$ such that

$$
\begin{aligned}
& \Psi\left(t_{1}\right)=0, \quad \dot{\Psi}\left(t_{1}\right) \geqslant 0 \\
& \Psi(t)<0, \quad h \leqslant t \leqslant t_{1} .
\end{aligned}
$$

For $\tau>0$, if $h \leqslant t_{1}-\tau \leqslant t_{1}$, it follows from (25) that

$$
P_{0}\left(t_{1}-\tau\right)<\beta \bar{Q}_{0} \mathrm{e}^{a_{2}\left(t_{1}-h\right)} \leqslant \beta \bar{Q}_{1} \mathrm{e}^{a_{2}\left(t_{1}-h\right)} .
$$

And if $-\tau \leqslant t_{1}-\tau<h$, from (19) and (20), we have

$$
P_{0}\left(t_{1}-\tau\right) \leqslant P_{1}\left(t_{1}-\tau\right)<\beta \bar{Q}_{1} \leqslant \beta \bar{Q}_{1} \mathrm{e}^{a_{2}\left(t_{1}-h\right)} .
$$

Hence, for $\tau>0$, we always have

$$
P_{0}\left(t_{1}-\tau\right)<\beta \bar{Q}_{1} \mathrm{e}^{a_{2}\left(t_{1}-h\right)} .
$$

Then,

$$
\begin{aligned}
\dot{\Psi}\left(t_{1}\right) & =\lambda P_{0}\left(t_{1}\right)+\mathrm{e}^{\lambda t_{1}} \dot{V}_{0}\left(t_{1}\right)- \\
& a_{2} \beta \bar{Q}_{0} \mathrm{e}^{a_{2}\left(t_{1}-h\right)} \leqslant \\
& \lambda P_{0}\left(t_{1}\right)+\left(a_{2}-a_{1}\right) \mathrm{e}^{\lambda t_{1}} V_{0}\left(t_{1}\right)+ \\
& \frac{N \bar{w}_{1}^{2}}{q} \mathrm{e}^{\lambda t_{1}} V_{0}\left(t_{1}-\tau\right)- \\
& a_{2} \beta \bar{Q}_{0} \mathrm{e}^{a_{2}\left(t_{1}-h\right)} \leqslant \\
& \left(\lambda+a_{2}-a_{1}\right) P_{0}\left(t_{1}\right)+\frac{N \bar{w}_{1}^{2}}{q} \mathrm{e}^{\lambda \tau} P_{0}\left(t_{1}-\tau\right)- \\
& a_{2} \beta \bar{Q}_{0} \mathrm{e}^{a_{2}\left(t_{1}-h\right)}< \\
& \left(\lambda-a_{1}+\frac{N \bar{w}_{1}^{2}}{q} \mathrm{e}^{\lambda \tau}\right) \beta \bar{Q}_{0} \mathrm{e}^{a_{2}\left(t_{1}-h\right)}=0
\end{aligned}
$$

which contradicts the second inequality in (24). Hence, $\Psi(t)<0$ holds, i.e., for $t \in[h, T)$, we have

$$
\begin{aligned}
P_{0}(t) & <\beta \bar{Q}_{0} \mathrm{e}^{a_{2}\left(t_{1}-h\right)} \leqslant \beta \bar{Q}_{0} \mathrm{e}^{a_{2}(T-h)}< \\
& \beta \bar{Q}_{1} \mathrm{e}^{a_{2}(T-h)} .
\end{aligned}
$$

On the other hand, it follows from (19) and (20) that for $t \in[-\tau, h)$

$$
P_{0}(t)<\beta \bar{Q}_{1}<\beta \bar{Q}_{1} \mathrm{e}^{a_{2}(T-h)} .
$$

So,

$$
P_{0}(t)<\beta \bar{Q}_{1} \mathrm{e}^{a_{2}(T-h)}, \quad \text { for } \quad \text { all } t \in[-\tau, T) .
$$

Similarly, we can prove that for $t \in[T, h+T), P_{0}(t)<$ $\beta \bar{Q}_{1} \mathrm{e}^{a_{2}(T-h)}$, and for $t \in[T+h, 2 T), P_{0}(t)<\beta \bar{Q}_{1} \mathrm{e}^{a_{2}(T-2 h)}$.

By induction, we can derive the following estimation of $P_{0}(t)$ for any integer $\kappa$.

For $\kappa T \leqslant t<\kappa T+h, \kappa=0,1,2, \cdots$,

$$
P_{0}(t)<\beta \bar{Q}_{1} \mathrm{e}^{a_{2} \kappa(T-h)} \leqslant \beta \bar{Q}_{1} \mathrm{e}^{a_{2}\left(1-\frac{h}{T}\right) t} .
$$

And for $\kappa T+h \leqslant t<(\kappa+1) T, \kappa=0,1,2, \cdots$,

$$
P_{0}(t)<\beta \bar{Q}_{1} \mathrm{e}^{a_{2}[t-(\kappa+1) h]} \leqslant \beta \bar{Q}_{1} \mathrm{e}^{a_{2}\left(1-\frac{h}{T}\right) t} .
$$

Letting $\beta \rightarrow 1$, from the definition of $P_{0}(t)$, we obtain

$$
\begin{aligned}
V_{0}(t) \leqslant & \bar{Q}_{1} \exp \left\{-\left[\lambda-a_{2}\left(1-\frac{h}{T}\right) t\right]\right\}= \\
& \sup _{-\tau \leqslant s \leqslant 0} V_{1}(s) \mathrm{e}^{-\varepsilon t} \quad t \geqslant 0 .
\end{aligned}
$$


It follows from the condition in the third inequality of (10) that the zero solution of the error dynamical system (9) is globally exponentially stable.

Remark 1. Xia et al. ${ }^{[17,19]}$ studied pinning synchronization of complex network with delayed dynamical nodes via linear periodically intermittent control. The control gains obtained in $[17,19]$ are larger than the needed values for practical problems. But in our results, we adopt the adaptive pinning control approach and give a rigorous proof for the synchronization scheme with the adaptive controller. Thus, our results are less conservative and more practically applicable.

If $\sigma=2 L+N b^{2} q+2 \bar{w}_{0}^{2} \lambda_{\max }\left(\hat{A}^{s}\right)$ and $a_{2}=a_{1}+\sigma>0$, then the third inequality in Theorem 1 holds. Thus, we can have the following corollary.

Corollary 1. Under Assumption 1, if there exist positive constants $\varepsilon, a_{1}$, and $\alpha_{i}$, such that

$$
\begin{aligned}
& a_{1}>\frac{N \bar{w}_{1}^{2}}{q} \\
& \lambda_{\max }\left(\overline{\hat{A}}^{s}\right) \leqslant-\frac{2 L+N b^{2} q}{\bar{w}_{0}^{2}} \\
& \varepsilon=\lambda-\left(a_{1}+\sigma\right)\left(1-\frac{h}{T}\right)>0
\end{aligned}
$$

where $\lambda>0$ is the smallest real root of $a_{1}-\lambda-\frac{N \bar{w}_{1}^{2}}{q} \mathrm{e}^{\lambda \tau}=0$, then the controlled delayed complex networks (1) globally exponentially synchronize with the desired evolution $s(t)$ under the adaptive pinning periodically intermittent controllers (7) and the updating laws (8).

\subsection{Exponential synchronization of de- layed complex dynamical networks with different nodes}

Because all the subsystems are the same system in the complex networks (1), the scope of application is restricted. Then we will give the exponential synchronization criterion of delayed complex dynamical networks with different nodes via the adaptive pinning periodically intermittent controllers.

Consider that the delayed complex dynamical networks with different nodes can be described as

$$
\begin{aligned}
\dot{x}_{i}(t)= & f_{i}\left(x_{i}(t), t\right)+\sum_{j=1}^{N} a_{i j} H_{0}\left(x_{j}(t)\right)+ \\
& \sum_{j=1}^{N} b_{i j} H_{1}\left(x_{j}(t-\tau)\right)+u_{i}(t)
\end{aligned}
$$

where $f_{i}(\cdot),(i=1, \cdots, N): \mathbf{R}^{n} \times \mathbf{R}^{+} \rightarrow \mathbf{R}^{n}$ are the continuously differentiable vector functions, the other parameters are the same as those in (1).

Assumption 2. There exist positive constants $M_{i}(i=$ $1,2, \cdots, N), \underline{w}_{l}, \bar{w}_{l}(l=0,1)$ satisfying

$$
\begin{aligned}
& \left\|f_{i}\left(x_{i}(t), t\right)-f(s(t), t)\right\| \leqslant M_{i}\left\|x_{i}(t)-s(t)\right\| \\
& \underline{w}_{l}\left\|e_{i}\left(t-\tau_{l}\right)\right\| \leqslant\left\|W_{l}\left(e_{i}\left(t-\tau_{l}\right)\right)\right\| \leqslant \bar{w}_{l}\left\|e_{i}\left(t-\tau_{l}\right)\right\|
\end{aligned}
$$

where $x_{i}(t)$ are time-varying vectors, and $s(t)$ is a solution of an isolate node.
According to the same method of Theorem 1 and based on Assumption 2, it is easy to obtain the synchronization criterion for the controlled delayed complex networks with different nodes (32) as follows.

Theorem 2. Under Assumption 2, if there exist positive constants $a_{1}>\frac{N \bar{w}_{1}^{2}}{q}$ and $\alpha_{i}$, such that

$$
\begin{aligned}
& \lambda_{\max }\left(\overline{\hat{A}}^{s}\right) \leqslant-\frac{2 M+N b^{2} q}{\bar{w}_{0}^{2}} \\
& \lambda>\left(a_{1}+\sigma\right)\left(1-\frac{h}{T}\right)
\end{aligned}
$$

where $M=\max \left\{M_{1}, \cdots, M_{N}\right\}$ and $\lambda$ is the smallest real root of $a_{1}-\lambda-\frac{N \bar{w}_{1}^{2}}{q} \mathrm{e}^{\lambda \tau}=0$, then the controlled delayed complex networks with different nodes (32) globally exponentially synchronize with the desired evolution $s(t)$ under the adaptive pinning periodically intermittent controllers (7) and the updating laws (8).

\section{Simulation example}

In this section, we use a representative example to illustrate the results derived in this work. Here, we consider the delayed complex networks that consist of 10 identical Chen systems $^{[24]}$ described as

$$
\begin{aligned}
\dot{x}_{i}(t)= & f\left(x_{i}(t), t\right)+\sum_{j=1}^{10} a_{i j} H_{0}\left(x_{j}(t)\right)+ \\
& \sum_{j=1}^{10} b_{i j} H_{1}\left(x_{j}(t-\tau)\right)+u_{i}(t)
\end{aligned}
$$

where $i=1,2, \cdots, 10$. The asymmetric outer coupling matrices $A$ and $B$ are random and satisfy the coupling condition, i.e., $a_{i i}=-\sum_{j=1, j \neq i}^{N} a_{i j}, b_{i i}=-\sum_{j=1, j \neq i}^{N} b_{i j}$. The nonlinear inner coupling function is continuous and has the form: $\left.H_{0}\left(x_{j}(t)\right)=\left(x_{i 1}^{2}(t), 2 x_{i 2}(t) x_{i 3}(t), x_{i 3}(t)\right)\right)$, $H_{1}\left(x_{j}(t-\tau)\right)=\left(\sin \left(x_{i 1}(t-\tau)\right), x_{i 2}(t-\tau), \arctan \left(x_{i 3}(t-\tau)\right)\right)$, $\tau=0.04$. The dynamics of the Chen oscillator $f\left(x_{i}(t), t\right)$ is given by ${ }^{[24]}$

$$
\left\{\begin{array}{l}
\dot{x}_{i 1}(t)=35\left(x_{i 2}(t)-x_{i 1}(t)\right) \\
\dot{x}_{i 2}(t)=-7 x_{i 1}(t)-x_{i 1}(t) x_{i 3}(t)+28 x_{i 2}(t) \\
\dot{x}_{i 3}(t)=x_{i 1}(t) x_{i 2}(t)-3 x_{i 3}(t) .
\end{array}\right.
$$

Since the chaotic attractor is bounded in a certain region, there exists a constant $M$ satisfying $\left\|s_{i k}\right\|<R=30.9342$ for $1 \leqslant i \leqslant 10,1 \leqslant k \leqslant 3^{[14]}$. Then,

$$
\begin{aligned}
& \left\|f\left(x_{i}(t), t\right)-f(s(t), t)\right\|= \\
& \quad \sqrt{\left[-x_{i 3} e_{i 1}-s_{1} e_{i 3}\right]^{2}+\left[x_{i 2} e_{i 1}+s_{1} e_{i 2}\right]^{2}} \leqslant 2 R\left\|e_{i}\right\| .
\end{aligned}
$$

Obviously, the Chen system and nonlinear inner coupling functions $H_{l}(\cdot)$ satisfy Assumption 1.

In this example, it is supposed that the desired exponential synchronization rate $\varepsilon=1.1$. The values of the parameters for the controllers (7) and the updating laws (8) are taken as $T=0.2, h=0.15, \alpha_{i}=5(i=1,2,3,4), \alpha_{i}=$ $0(i=5, \cdots, 10)$ (i.e., we only pin and control four nodes of the complex network), and $q=1$. If we choose $a_{1}=30$ 
and $a_{2}=48$, it is easy to verify that $(10)$ in Theorem 1 is satisfied. According to Theorem 1, one can synchronize the network (32) by designing the following adaptive controllers $(7)$ and $(8)$, where $s(0)=(1,2,1)^{\mathrm{T}}$. Fig. 1 shows the synchronous errors $e_{i 1}, e_{i 2}, e_{i 3}(i=1,2, \cdots, 10)$ of the delayed complex network under the adaptive pinning periodically intermittent feedback controllers. Obviously, the synchronous solution is exponential stable for the delayed complex dynamical network (35) under the adaptive pinning periodically intermittent feedback controllers (7) and updating laws (8). Moreover, the values for the control gains which are obtained in Theorem 1 after synchronization satisfy $k_{i} \leqslant 12.2$. But if we adopt the linear periodically intermittent control which is obtained in $[17,19]$, the values for the synchronization control gains satisfy $k_{i} \leqslant 74.46$. It is illustrated that the adaptive control approach can obtain a more applicable and smaller control gain.
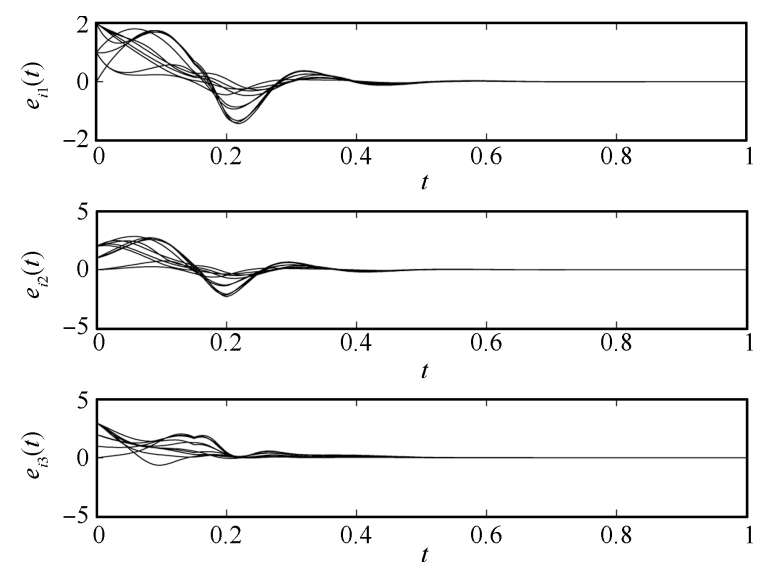

Fig. 1 Synchronization errors $e_{i j}(i=1,2, \cdots, 10, j=1,2,3)$ of complex network (32) under the adaptive pinning periodically intermittent feedback controllers (7) and (8).

\section{Conclusions and discussions}

In this paper, the problem of synchronization for a class of general complex dynamical networks with nonlinear coupling delays under the adaptive pinning periodically intermittent control has been investigated in detail. Based on the piecewise Lyapunov function combined with periodically intermittent control technique, the method of adaptive control and pinning control, some general criteria for ensuring delayed complex dynamical networks synchronization have been derived. And several corresponding adaptive pinning feedback synchronization controllers are designed. Moreover, the traditional assumptions on control width and time delays are released in our results. Finally, numerical simulation has verified the effectiveness of the presented method.

There are some interesting discussions to the relationship about the synchronization of complex network. In essence, chaos control is a special case of chaos synchronization, and it is considered to achieve synchronization with $O(0,0,0)$. Synchronization of fractional-order chaotic system is a broad concept of synchronization of integerorder chaotic systems. In other words, the fractionalorder chaotic system is an integer-order system if the order of fractional-order system is $q=1$. For the complex network in which the nodes are integer-order chaotic system or fractional-order chaotic system, the synchronization which uses adaptive pinning periodically intermittent control should be studied. Moreover, synchronization and antisynchronization are the same concept in essence. We will design some novel controllers in our future work, by which both synchronization and anti-synchronization of complex network can be achieved.

\section{References}

[1] R. Albert, A. L. Barabási. Statistical mechanics of complex networks. Reviews of Modern Physics, vol. 74, no. 1, pp. 4797, 2002.

[2] S. H. Strogatz. Exploring complex networks. Nature, vol. 410, no. 6825 , pp. 268-276, 2001.

[3] X. F. Wang, G. R. Chen. Complex networks: Small-world, scale-free and beyond. IEEE Circuits and Systems Magazine, vol. 3, no. 1, pp. 6-20, 2003.

[4] J. H. Lü, X. H. Yu, G. R. Chen. Chaos synchronization of general complex dynamical networks. Physica A: Statistical Mechanics and its Applications, vol. 334, no.1-2, pp. 281302, 2004.

[5] T. L. Liao, S. H. Tsai. Adaptive synchronization of chaotic systems and its application to secure communications. Chaos, Solitons \& Fractals, vol.11, no. 9, pp. 1387-1396, 2000.

[6] Z. D. Wang, Y. Wang, Y. R. Liu. Global synchronization for discrete-time stochastic complex networks with randomly occurred nonlinearities and mixed time delays. IEEE Transactions on Neural Networks, vol. 21, no. 1, pp. 11-25, 2010.

[7] Y. Dai, Y. Z. Cai, X. M. Xu. Synchronization and exponential estimates of complex networks with mixed time-varying coupling delays. International Journal of Automation and Computing, vol. 6, no. 3, pp. 301-307, 2009.

[8] H. Y. Sun, N. Li, H. Sun, H. Niu. Adaptive synchronization control of the delayed complex dynamical networks and its application on electromechanical systems. ICIC Express Letters, vol. 5, no. 10, pp. 3605-3611, 2011.

[9] J. D. Cao, P. Li, W. W. Wang. Global synchronization in arrays of delayed neural networks with constant and delayed coupling. Physics Letters A, vol. 353, no. 4, pp. 318$325,2006$.

[10] J. Zhou, J. A. Lu, J. H. Lü. Adaptive synchronization of an uncertain complex dynamical network. IEEE Transactions on Automatic Control, vol.51, no. 4, pp.652-656, 2006.

[11] W. L. Guo, M. Z. Mao. Projective lag synchronization and parameter identification of a new hyperchaotic system. International Journal of Automation and Computing, vol. 10, no. 3, pp. 256-259, 2013.

[12] H. Y. Sun, Q. L. Zhang, N. Li. Synchronization control of united complex dynamical networks with multi-links. International Journal of Innovative Computing, Information and Control, vol. 7, no. 2, pp. 927-939, 2011.

[13] Y. Dai, Y. Cai, X. Xu. Synchronisation analysis and impulsive control of complex networks with coupling delays. IET Control Theory \& Applications, vol. 3, no. 9, pp. 1167-1174, 2009.

[14] W. W. Yu, G. R. Chen, J. H. Lü. On pinning synchronization of complex dynamical networks. Automatica, vol. 45, no. 2, pp. 429-435, 2009.

[15] X. S. Yang, J. D. Cao. Stochastic synchronization of coupled neural networks with intermittent control. Physics Letters A, vol. 373, no. 36, pp. 3259-3272, 2009. 
[16] W. G. Xia, J. D. Cao. Pinning synchronization of delayed dynamical networks via periodically intermittent control. Chaos, vol. 19, no. 1, 013120, 2009.

[17] S. M. Cai, Z. R. Liu, F. D. Xu, J. W. Shen. Periodically intermittent controlling complex dynamical networks with time-varying delays to a desired orbit. Physics Letters A, vol. 373, no. 42, pp. 3846-3854, 2009.

[18] S. M. Cai, J. J. Hao, Q. B. He, Z. R. Liu. Exponential synchronization of complex delayed dynamical networks via pinning periodically intermittent control. Physics Letters A, vol. 375, no. 19, pp. 1965-1971, 2011.

[19] D. Y. Chen, L. Y. Xiao, X. Y. Ma, R. F. Zhang. Control of a class of fractional-order chaotic systems via sliding mode. Nonlinear Dynamics, vol. 67, no. 1, pp. 893-901, 2012.

[20] D. Y. Chen, R. F. Zhang, X. Y. Ma, S. Liu. Chaotic synchronization and anti-synchronization for a novel class of multiple chaotic systems via a sliding mode control scheme. Nonlinear Dynamics, vol. 69, no. 1-2, pp. 35-55, 2012.

[21] D. Y. Chen, R. F. Zhang, J. C. Sprott, H. T. Chen, X. Y. Ma. Synchronization between integer-order chaotic systems and a class of fractional-order chaotic systems via sliding mode control. Chaos, vol. 22, no. 2, 023130, 2012.

[22] D. Y. Chen, W. L. Zhao, J. C. Sprott, X. Y. Ma. Application of Takagi-Sugeno fuzzy model to a class of chaotic synchronization and anti-synchronization. Nonlinear Dynamics, 2013. (Online first).

[23] M. Zochowski. Intermittent dynamical control. Physica D: Nonlinear Phenomena, vol. 145, no. 3-4, pp. 181-190, 2000.

[24] G. R. Chen, T. Ueta. Yet another chaotic attractor. International Journal of Bifurcation and Chaos, vol. 9, no. 7, pp. 1465-1466, 1999.

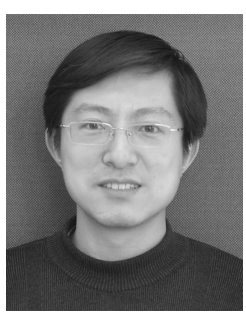

Hai-Yi Sun received the B. Sc. degree in mathematics from Shenyang Normal University, China in 2000, the M. Sc. degree in operational research and cybernetics, and the Ph. D. degree in control theory and control engineering, from Northeastern University, China in 2006 and 2011. He is an associate professor of Shenyang Jianzhu University, China. He has published about 30 refereed journal and conference papers.

His research interests include nonlinear control, chaos and synchronization control and complex networks.

\section{E-mail: shy_xx@163.com}

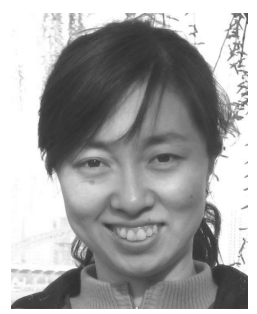

Ning Li received the B.Sc. and M. Sc. degrees in mathematics from Liaoning Normal University, China in 2001 and 2004, respectively, and the $\mathrm{Ph}$. D. degree in control theory and control engineering from Northeastern University, China in 2010. She is a lecturer of Northeastern University, China She has published about 30 refereed journal and conference papers.

Her research interests include bifurcations, chaos and the control in biological systems and complex networks.

E-mail: lining80@163.com (Corresponding author)

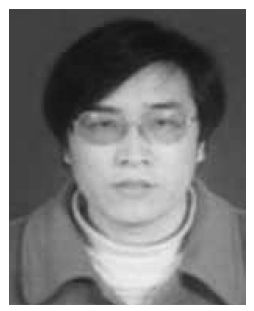

De-Ping Zhao received the B.Sc. and M. Sc. degrees from Mathematics Department, Nankai University, China in 1983, the M. Sc. degree from Mathematics Department of Zhejiang University, China in 1991 and the $\mathrm{Ph} . \mathrm{D}$. degree in computer theory and software engineering from Northeastern University, China in 2001. He is a professor of Shenyang Jianzhu University, China. He has published about 40 refereed journal and conference papers.

His research interests include chaos control, networked control system and image processing.

E-mail: zhaodeping@mail.sjzu.edu.cn

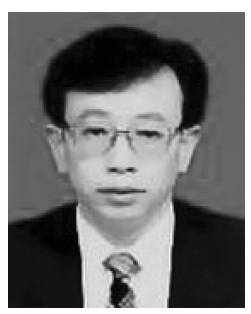

Qing-Ling Zhang received the B.Sc. and M. Sc. degrees from Mathematics Department, Northeastern University, China, and the Ph. D. degree from Automatic Control Department, Northeastern University, China in 1982, 1986, and 1995, respectively. $\mathrm{He}$ is a professor of Northeastern University, China. He is a member of University Teaching Advisory Committee of National Ministry of Education and Chairman of Liaoning Mathematical Society. He has published about 300 refereed journal and conference papers.

His research interests include chaos control, robust control, networked control system and singular system.

E-mail: qlzhang@mail.neu.edu.cn 